\title{
Two cases of response to pembrolizumab in epidermal growth factor receptor mutated lung adenocarcinoma patients with programmed death-ligand 1 overexpression
}

\author{
Takeshi Uenami, Mikako Ishijima, Masaki Kanazu, Hiroyuki Kurebe, Ryuya Edahiro, Kohei Nishida, \\ Yuki Akazawa, Yukihiro Yano, Toshihiko Yamaguchi, Masahide Mori \\ Department of Thoracic Oncology, National Hospital Organization Toneyama National Hospital, Osaka, Japan \\ Correspondence to: Takeshi Uenami, MD. Department of Thoracic Oncology, National Hospital Organization Toneyama National Hospital, 5-1-1 \\ Toneyama, Toyonaka City, Osaka 560-8552, Japan. Email: tuenami@toneyama.go.jp.
}

\begin{abstract}
Recently, the immune checkpoint inhibitor (ICI) pembrolizumab was demonstrated to be superior to platinum doublet chemotherapy in the first-line setting in patients with tumor programmed death-ligand 1 (PD-L1) expression of at least 50\%. However, because patients with epidermal growth factor receptor (EGFR) mutations or anaplastic lymphoma kinase (ALK) rearrangements were not included in that study, the efficacy of pembrolizumab in lung cancers carrying EGFR mutations could not be determined. Here we describe two cases of response to pembrolizumab in EGFR mutated lung adenocarcinoma patients with PD-L1 overexpression. These cases indicate that ICI is an effective treatment for EGFR mutated lung adenocarcinoma patients with PD-L1 overexpression.
\end{abstract}

Keywords: Non-small cell lung cancer (NSCLC); epidermal growth factor receptor (EGFR); pembrolizumab; programmed death-ligand 1 (PD-L1)

Submitted Jun 24, 2018. Accepted for publication Oct 10, 2018.

doi: $10.21037 /$ atm.2018.10.24

View this article at: http://dx.doi.org/10.21037/atm.2018.10.24

\section{Introduction}

Pembrolizumab, a monoclonal antibody targeting programmed cell-death 1 (PD-1), is effective for some advanced tumors including non-small cell lung cancer (NSCLC). In the KEYNOTE-024 phase 3 clinical trial, pembrolizumab led to the significant prolongation of overall survival compared with first-line platinumbased chemotherapy in NSCLC patients whose tumors expressed a programmed death-ligand 1 (PD-L1) tumor proportion score (TPS) of at least 50\% (1). In clinical practice, the use of pembrolizumab is rapidly increasing and immunohistochemical analysis of PD-L1 expression by tumor cells is being used as a predictive biomarker for the efficacy of this drug in the treatment of NSCLC.

However, in the KEYNOTE-024 study patients with epidermal growth factor receptor (EGFR) mutation or anaplastic lymphoma kinase (ALK) rearrangements were excluded. Therefore, the role of pembrolizumab in EGFR- mutated lung cancers remains unclear. Here, we report two cases of response to pembrolizumab in patients with EGFR mutated lung adenocarcinoma with PD-L1 overexpression.

\section{Case presentation}

Case 1

A 75 -year-old man with a 55-pack-year history of smoking was referred to our hospital because of an abnormal shadow in the right lung, which was detected by routine lung radiography in July 2017. A chest computed tomography (CT) scan showed a tumor of approximately $56-\mathrm{mm}$ diameter in the right upper lung with right mediastinal lymphadenopathy (Figure $1 A$ ). Positron emission tomography (PET) scanning revealed multiple metastases on the right adrenal gland, pancreas (Figure 1B), and mesentery (Figure 1C). A transbronchial lung biopsy was performed, and immunohistochemical staining revealed the 
Page 2 of 5
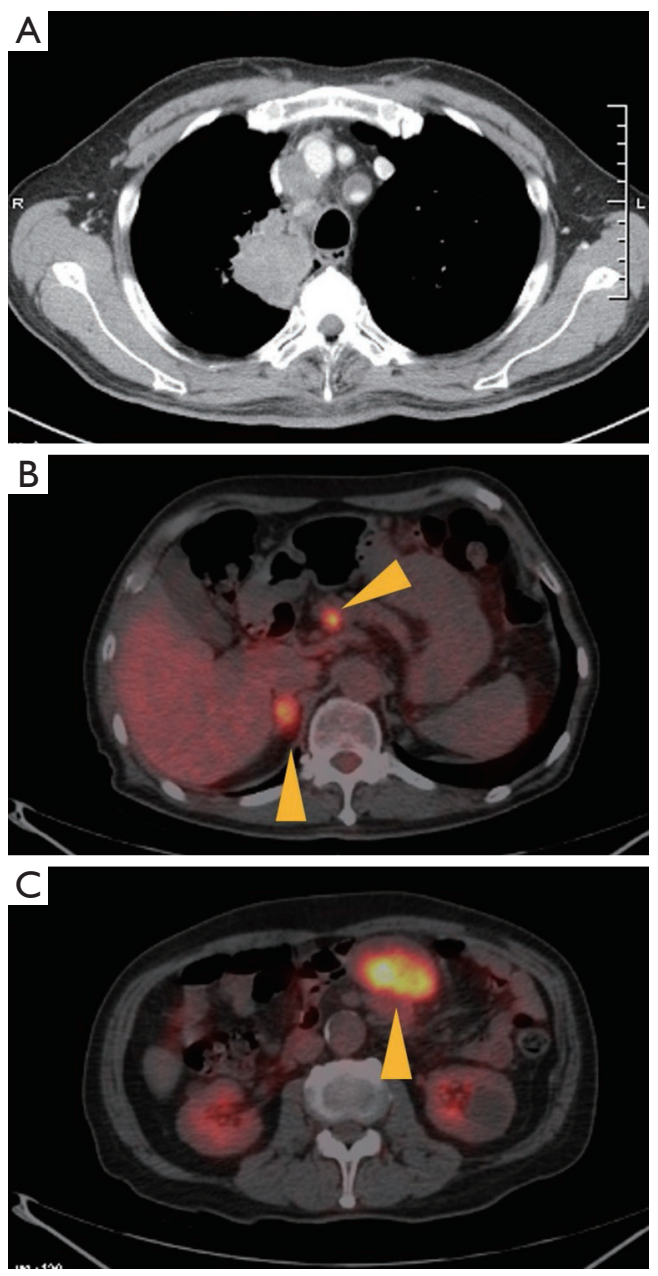

Figure 1 Baseline chest computed tomography scan and PET scan of patient in case 1. (A) The diameter of the tumor in the right upper lung was approximately $56 \mathrm{~mm}$, and mediastinal lymphadenopathy was observed. Accumulation of FDG on (B) the right adrenal gland, pancreas and $(C)$ mesentery indicated the presence of metastasis. PET, positron emission tomography; FDG, fluorodeoxyglucose.

tumor cells were positive for thyroid transcription factor-1 (TTF-1) and negative for $\mathrm{p} 40$; therefore, the patient was diagnosed as having adenocarcinoma. The tumors also had EGFR exon21 L858R point mutation [observed using peptide nucleic acid-locked nucleic acid polymerase chain reaction (PNA-LNA PCR) clamp method (LSI Medience)] and a PD-L1 TPS of almost 100\% [observed using immunohistochemistry clone $22 \mathrm{C} 3$ pharmDx assay (Dako)] (Figure 2). ALK or ROS1 rearrangements were not detected. The patient had stage IVB cancer (cT3N2M1c),

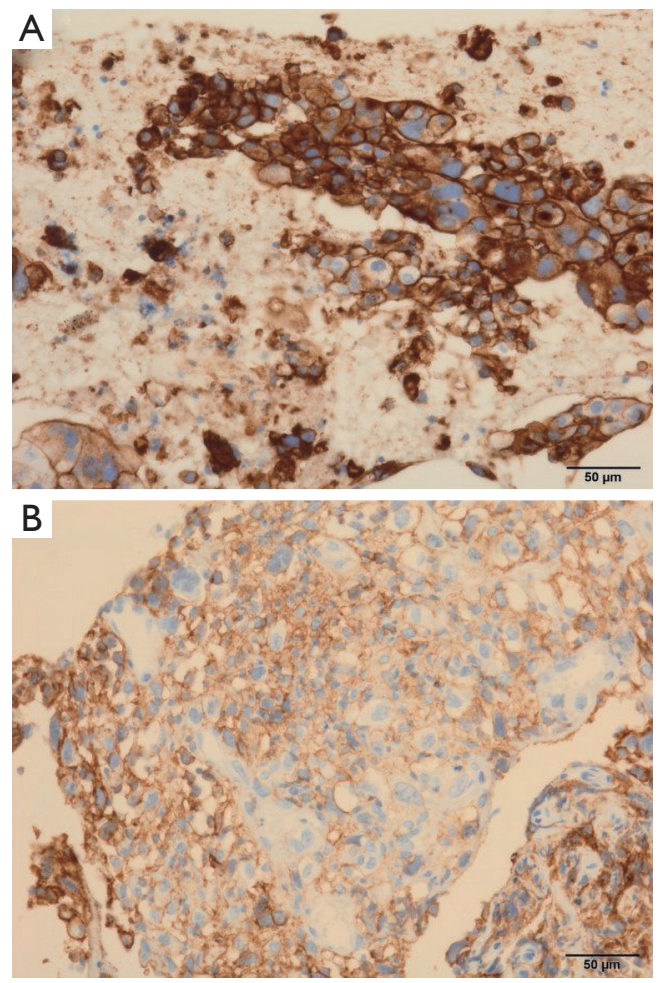

Figure 2 PD-L1 TPS immunohistochemistry analysis $(\times 200$ magnification) in cases 1 and 2 using clone 22C 3 showed (A) almost $100 \%$ and (B) at least $95 \%$, respectively. PD-L1, programmed death-ligand 1; TPS, tumor proportion score.

and first-line treatment with erlotinib was started in August 2017.

In the first 21 days after treatment with erlotinib, the patient experienced abdominal pain. Laboratory findings revealed that serum amylase and lipase levels were increased. A CT scan demonstrated mainly pancreatic tail edema with infiltration of peripancreatic fat, no sign of necrosis, and an increase in the size of mesenteric metastasis. The patient was diagnosed with acute pancreatitis and had no response to erlotinib (Figure $3 A$ ). In order to address the pancreatitis, massive hydration and antibiotics were administered. On day 27 , serum amylase levels improved to almost normal range and the abdominal pain was resolved. At this juncture, pembrolizumab was planned as second-line therapy.

Pembrolizumab was administered at a standard dosage (200 mg, triweekly). Two days after treatment with pembrolizumab, the mesenteric metastasis ruptured into the peritoneal cavity and conservative management was undertaken since surgery was considered high risk. Twelve 

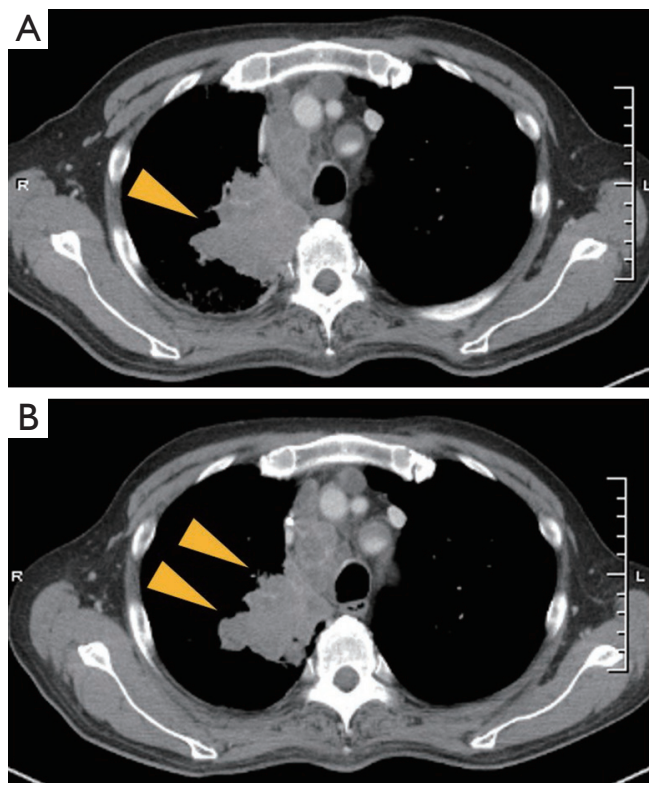

Figure 3 Radiographical changes of the patient in case 1 during the pembrolizumab treatment (A) after treatment with erlotinib, CT scan showed a greater increase in the size of tumor in the right upper lobe and mediastinal lymphadenopathy, compared with the size shown in the initial CT scan; (B) twenty-one days after beginning pembrolizumab treatment, CT scan showed a reduced tumor size in the primary lesion of the right lung. CT, computed tomography.

days after rupture, the patient was further diagnosed with a catheter-related bloodstream infection. Twenty-one days after beginning pembrolizumab treatment, a CT scan showed reduced tumor size in the primary lesion of the right lung (Figure 3B) and stable mesenteric metastasis. His target lesions were decreased $(-13 \%)$ according to the definition of the Response Evaluation Criteria in Solid Tumors (RECIST) v1.1; therefore, treatment with pembrolizumab was considered to have resulted in stable disease (SD). Treatment was achieved through 2 cycles, however pembrolizumab was discontinued due to disseminated intravascular coagulation.

\section{Case 2}

An 83-year-old woman with a 22-pack-year history of smoking was referred to our hospital because of bloody sputum in November 2016. Chest CT scanning showed a tumor of approximately $28-\mathrm{mm}$ diameter in the left upper lung with pleural effusion (Figure $4 A$ ). The patient was
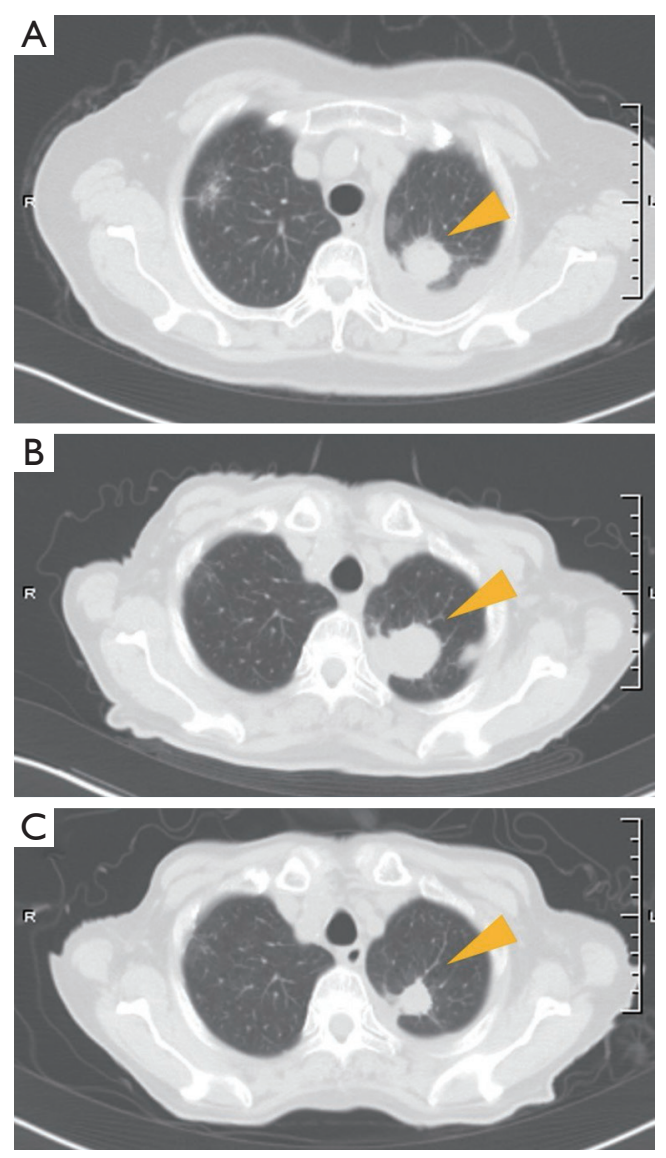

Figure 4 Radiographically changes of the patient in case 2 during the treatment (A) CT scan showed a tumor of approximately $28 \mathrm{~mm}$ diameter in the left upper lung with pleural effusion; (B) 11 months after treatment with gefitinib, a CT scan revealed disease progression of the lung lesion; (C) 1.5 months after beginning pembrolizumab treatment, CT scan revealed a partial response: a reduced tumor size in the primary lesion. CT, computed tomography.

diagnosed with stage IVA adenocarcinoma (cT2aN0M1a) with EGFR Exon 19 deletion mutation, as analyzed using a sample from the pleural effusion. Immunohistochemically, the tumor cells were positive for TTF-1 and negative for p40. First-line treatment with gefitinib was initiated in December 2016. She continued gefitinib therapy with a partial response, however, after 11 months a CT scan revealed disease progression of the lung lesion (Figure $4 B$ ). According to findings of a transbronchial lung re-biopsy, the patient had a mutation of EGFR in the form of an Exon19 deletion and a PD-L1 TPS of at least 95\% (Figure 2), but was negative for the Exon20 T790M mutation [observed 
using the cobs EGFR mutation test (Roche Molecular Systems)]. Pembrolizumab was planned as second-line therapy in March 2018. After 1.5 months, a CT scan showed the primary lesion of left upper lung was decreased $(-44 \%)$ according to the RECIST v1.1 and revealed a partial response (Figure $4 C$ ). Thus, treatment with pembrolizumab is ongoing.

\section{Discussion}

Lung cancer is the leading cause of cancer-related death worldwide (2). Recently, immune checkpoint inhibitors (ICIs) have been investigated as a new treatment agent and treatment strategies for patients with advanced NSCLC have expanded. Currently, chemotherapy, ICIs, and molecular target therapy are used, singly or in combination, for the treatment of this disease.

The optimal treatment for cases of advanced NSCLC is determined using the results of PD-L1 TPS and targeted somatic genomic analysis (EGFR mutation, ALK rearrangement). This approach is taken because pembrolizumab has shown a remarkable response in patients with PD-L1 overexpression, while molecularly targeted drugs such as EGFR-tyrosine kinase inhibitor (TKI) and AKI-TKI greatly improve the clinical outcome of advanced NSCLC patients with sensitive EGFR gene mutations (3) or ALK gene translocations, respectively.

EGFR-TKI is a key drug for the treatment of NSCLC patients with EGFR mutations, however the role of ICIs in EGFR-mutated lung cancer is controversial. A meta-analysis revealed that ICIs were less effective for the treatment of NSCLC with EGFR mutations than for lung tumors with wild-type EGFR (4). In a retrospective analysis of 58 patients with advanced NSCLC treated with PD-1/PD-L1 inhibitors, only $4 \%$ of patients harboring EGFR mutations or ALK rearrangements responded to ICI treatment, while $23 \%$ of patients with wild-type EGFR and ALK-negative or unknown tumors were responders (5). In addition, EGFR mutant NSCLC was less likely to be PD-L1 positive compared with wild-type EGFR mutant tumors (6). Only $11 \%$ of lung adenocarcinoma patients with EGFR mutations or ALK rearrangements have a PD-L1 TPS of at least $50 \%$ as measured with the $22 \mathrm{C} 3$ assay (7), which may explain the poor response of these tumors to ICI therapy.

In ATLANTIC, a phase 2, single-arm trial of thirdline and later durvalumab treatment in advanced NSCLC, patients with at least $25 \%$ of tumor cells expressing PD-L1 (assessed using SP263 Assay) had a longer median overall survival than patients with less than $25 \%$ of tumor cells expressing PD-L1, regardless of EGFR or ALK status (8). On the other hand, a previous report showed that the efficacy of PD-1/PD-L1 inhibitors seems to be low among patients with EGFR-mutated or ALK rearranged lung cancer, across all levels of tumor PD-L1 expression (5).

There are a number of potential reasons for this observed difference in response to ICI therapy in patients with or without EGFR or ALK mutations. First, PD-L1 expression may be dynamic within individual patients, with some exhibiting changes over time and in response to treatment. Recently, it was reported that about $20 \%$ of EGFR mutation-positive NSCLC patients demonstrated increased PD-L1 expression in post-TKI specimens compared to the pre-TKI specimens (5). Second, two different mechanisms of PD-L1 upregulation in tumors have been described: adaptive immune resistance, which refers to induction of tumor PD-L1 expression in response to local inflammatory signals produced by an active antitumor immune response; and innate immune resistance, which refers to upregulation of PD-L1 as a result of constitutive oncogenic signaling within cancer cells (9). It remains unclear whether, or which of, these two different mechanisms underlying tumor PD-L1 expression impacts responsiveness to PD-1/PD-L1 inhibitors (5). Finally, to examine PD-L1 expression, various antibodies such as clone SP263 or SP142 were used in previous reports. Differences across studies may reflect the use of different anti-PD-L1 antibodies and scoring cut-offs.

Currently, next-generation EGFR-TKI are used as first line therapy, and prioritization of therapies will be particularly important in choosing treatment strategies. Therefore, after the failure or 1st or 2nd generation EGFRTKI, it would be prudent to examine the presence of both T790M mutation and PD-L1 expression. In this report, we have determined that immunotherapy with PD-L1 inhibitors is an option as a second line therapy for advanced lung adenocarcinoma harboring EGFR mutation with PDL1 overexpression. Nevertheless, a larger prospective study is needed to assess these results.

\section{Acknowledgments}

We thank Dr. Koji Urasaki (Department of Laboratory Medicine and Pathology, National Hospital Organization Toneyama National Hospital) for his contribution in the performing of the immunohistochemical analysis. 


\section{Footnote}

Conflicts of Interest: The authors have no conflicts of interest to declare.

Informed Consent: Written informed consent was obtained from the patients for publication of this manuscript and any accompanying images.

\section{References}

1. Reck M, Rodriguez-Abreu D, Robinson AG, et al. Pembrolizumab versus Chemotherapy for PD-L1Positive Non-Small Cell Lung Cancer. New Engl J Med 2016;375:1823-33.

2. Siegel RL, Miller KD, Jemal A. Cancer Statistics, 2017. CA Cancer J Clin 2017;67:7-30.

3. Oxnard GR, Binder A, Jänne PA. New targetable oncogenes in non-small-cell lung cancer. J Clin Oncol 2013;31:1097-104.

4. Lee CK, Man J, Lord S, et al. Checkpoint Inhibitors

Cite this article as: Uenami T, Ishijima M, Kanazu M, Kurebe H, Edahiro R, Nishida K, Akazawa Y, Yano Y, Yamaguchi T, Mori M. Two cases of response to pembrolizumab in epidermal growth factor receptor mutated lung adenocarcinoma patients with programmed death-ligand 1 overexpression. Ann Transl Med 2018;6(22):444. doi: 10.21037/atm.2018.10.24 in Metastatic EGFR-Mutated Non-Small Cell Lung Cancer-A Meta-Analysis. J Thorac Oncol 2017;12:403-7.

5. Gainor JF, Shaw AT, Sequist LV, et al. EGFR Mutations and ALK Rearrangements Are Associated with Low Response Rates to PD-1 Pathway Blockade in Non-Small Cell Lung Cancer: A Retrospective Analysis. Clin Cancer Res 2016;22:4585-93.

6. Soo RA, Lim SM, Syn NL, et al. Immune checkpoint inhibitors in epidermal growth factor receptor mutant non-small cell lung cancer: Current controversies and future directions. Lung Cancer 2018;115:12-20.

7. Yoneshima Y, Ijichi K, Anai S, et al. PD-L1 expression in lung adenocarcinoma harboring EGFR mutations or ALK rearrangements. Lung Cancer 2018;118:36-40.

8. Garassino MC, Cho BC, Kim JH, et al. Durvalumab as third-line or later treatment for advanced non-small-cell lung cancer (ATLANTIC): an open-label, single-arm, phase 2 study. Lancet Oncol 2018;19:521-36.

9. Pardoll DM. The blockade of immune checkpoints in cancer immunotherapy. Nat Rev Cancer 2012;12:252-64. 